\title{
The different release techniques in high level archery: a comparative case study
}

\author{
Deniz SIMSEK, Hayri ERTAN
}

Faculty of Sports Science, Anadolu University, Eskisehir, Turkey.

Address Correspondence to D. Simsek, ds@anadolu.edu.tr

\begin{abstract}
The muscle-contraction strategy between the predominant forearm and pull finger used in archery is defined as a response to the fall of the "clicker" by active contraction of the m. extensor digitorum (MED) and the gradual relaxation of the m. flexor digitorum superficialis (MFDS). However, there are no studies regarding whether different finger placement techniques on the bowstring have an effect on proper balance between the forearm flexor/extensor muscles. This different hook strategy (upper two fingers, lower two fingers, and three finger hook) may affect isometric contraction before the snap of the clicker, which is thought to have positive effects on archer performance (muscle activation and balance). The purpose of this study is to make a more detailed analysis of the different hook strategy performed by a particular top-level archer and to consider the advantages this strategy may have on bowstring behavior after release. A high-level archer (FITA scores > 1300) volunteered to participate in this study. The subject engaged in a single test session consisting of six shots. The electromyography (EMG) activity of the drawing arm muscle was quantified. The finger hook has affected isometric contraction before the snap of the clicker and caused sudden contraction of extensor, gradually relaxation of flexor muscles. The study results found that this three finger hook strategy can be used in the drawing arm with success, as it may avoid causing a lateral deflection of the bowstring.
\end{abstract}

Keywords: Archer, muscular contraction-relaxation, CoPsway.

\section{INTRODUCTION}

Archery can be described as a comparatively static sport requiring strength and endurance of the upper body, in particular the forearm and shoulder girdle (6). Skill in archery is defined as the ability to shoot an arrow to a given target in a certain time span with accuracy (5). The discipline is described as a six-phase movement. Each of these phases represents a stable sequence of movements and is ideal for studying motor control and skills acquired during this voluntary kinetic and kinematic process (8). An archer pushes the bow with an extended arm, which is statically held in the direction of the target, while the other arm exerts a dynamic pulling of the bowstring from the beginning of the drawing phase, until the release is dynamically executed (5). The release phase must be highly reproducible and well balanced to achieve the desired results in a competition (8). In particular, a repeated contraction and relaxation strategy in the forearm and pull finger muscles should be developed for this reason. There are some different hook techniques in archery such as the upper and lower two-finger hook. Because of those different hook approaches, the purpose of this study is to compare three different hook techniques in archery by using kinetic and kinematic methods.

\section{MATERIALS \& METHODS}

\section{Participants}

A high-level archer volunteered to participate in the study. Before taking the measurements, the subject was informed about the possible risks associated with the experiment and signed the informed consent. The participant was injury free at the time of testing and reported no previous injury history to his upper or lower limbs. The experimental procedures conformed to the Declaration of Helsinki and were approved by the local ethics committee.

\section{Measures}

The muscular activation values of extensor, flexor, deltoid (anterior-middle and posterior), trapezius (upper-middle and lower), and pectorals major muscles were compared in terms of the archer's three different hook techniques. The anterior-posterior $(\mathrm{fx})$ and medio-lateral (fy) force 
and center of pressure $(\mathrm{CoP})$ sway values during the successful shots at the goal from a distance of $18 \mathrm{~m}$ were analyzed. Before starting the test session, the participant performed a 15-minute warm-up without any interference by researchers for the shoulder girdle, elbow, and wrist and finger joints. The participant engaged in a single test session consisting of six shots. Prior to the shootings, the isometric maximum voluntary contraction (MVC) was obtained from M. flexor digitorum superficialis (MFDS), M. extensor digitorum communis (MEDC), M. deltoid anterior (MDA) M. deltoid middle (MDM), M. deltoid posterior (MDP), M. upper trapezius (MUT), M. middle trapezius (MMT), M. lower trapezius (MLT), M. pectoralis major (MPM) (9).

\section{Procedures}

The EMG activity of the nine muscles was recorded using surface electrodes (DELSYS Wireless Trigno Electromyography (EMG) 16-channel system). The band pass of the EMG amplifier, sampling rate, maximum intra-electrode impedance and CMMR were 20-500 Hz, $2000 \mathrm{~Hz}, 6 \mathrm{Ohms}$, and $95 \mathrm{~dB}$, respectively. The snap of the clicker triggered a 5V Transistor Logic (TTL) signal, which was registered simultaneously with the myoelectric signals. According to the rise of the TTL signal, muscular activation $400 \mathrm{~ms}$ before and $800 \mathrm{~ms}$ after were identified as pre-clicker and post-clicker intervals, respectively. The respective EMG data sets of each of the six shots were full-wave rectified and filtered (a moving filter with a 100ms time-window). During force measurement 600×400x100 mm "Kistler (Germany) 9281EA" force platform have used. Force data were sampled at $2000 \mathrm{~Hz}$ and normalized according to body weight. Data calculation formulas are given in Table 1. Data was analyzed using Bioware software. All data are expressed as mean \pm SD.

The force plate was placed $18 \mathrm{~m}$ away from the target. The imagery line from the center of the target to the shooting line was set at $90^{\circ}$, so that the $\mathrm{fx}$ direction represented the anterio-posterior sway and the fy direction represented the medio-lateral sway (Picture 1).

\section{Statistical Analysis}

Descriptive statistics were applied to identify the characteristics of a high-level archer. Mean scores were calculated for subject's 6 shots (All data are expressed as mean $\pm \mathrm{SD}$ ).

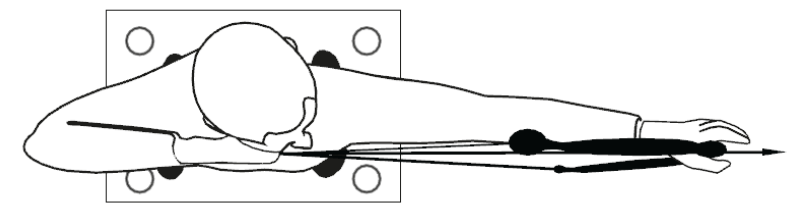

Channel

\begin{tabular}{|c|c|c|}
\hline Output Signal & Channel & Description \\
\hline $\mathrm{fx} 12$ & 1 & Force in X-direction measured by sensor $1+$ sensor 2 \\
\hline fx34 & 2 & Force in $\mathrm{X}$-direction measured by sensor $3+$ sensor 4 \\
\hline fy14 & 3 & Force in Y-direction measured by sensor $1+$ sensor 4 \\
\hline fy 23 & 4 & Force in Y-direction measured by sensor $2+$ sensor 3 \\
\hline $\mathrm{fz} 1 \ldots \mathrm{fz} 4$ & $5 \ldots 8$ & Force in $\mathrm{Z}$ direction measured by sensor $1 \ldots 4$ \\
\hline Parameter & Calculation & Description \\
\hline $\mathrm{Fx}$ & $=f \times 12+f \times 34$ & Medio-lateral force \\
\hline Fy & $=$ fy $14+$ fy 23 & Anterior-posterior force \\
\hline $\mathrm{Fz}$ & $=f z 1+f z 2+f z 3+f z 4$ & Vertical force \\
\hline Mx & $=\mathrm{b}^{*}(\mathrm{fz} 1+\mathrm{fz} 2-\mathrm{fz} 3-\mathrm{fz} 4)$ & Plate moment about $\mathrm{X}$-axis ${ }^{3)}$ \\
\hline \multirow[t]{2}{*}{ My } & $=\mathrm{a} *(-\mathrm{fz} 1+\mathrm{fz} 2+\mathrm{fz} 3-\mathrm{fz} 4)$ & Plate moment about $\mathrm{Y}$-axis ${ }^{3)}$ \\
\hline & $=b^{*}(-f \times 12+f \times 34)+a *(f y 14-f y 23)$ & Plate moment about $\mathrm{Z}$-axis ${ }^{3)}$ \\
\hline Mx1 & $=\mathrm{Mx}+\mathrm{Fy}^{*} \mathrm{az} 0$ & Plate moment about top plate surface ${ }^{2)}$ \\
\hline Ax & $=-\mathrm{My} 1 / \mathrm{Fz}$ & X-coordinate point of applied force $(\mathrm{CoP})^{2)}$ \\
\hline Ay & $=\mathrm{Mx} 1 / \mathrm{Fz}$ & Y-coordinate point of applied force $(\mathrm{CoP})^{2)}$ \\
\hline
\end{tabular}




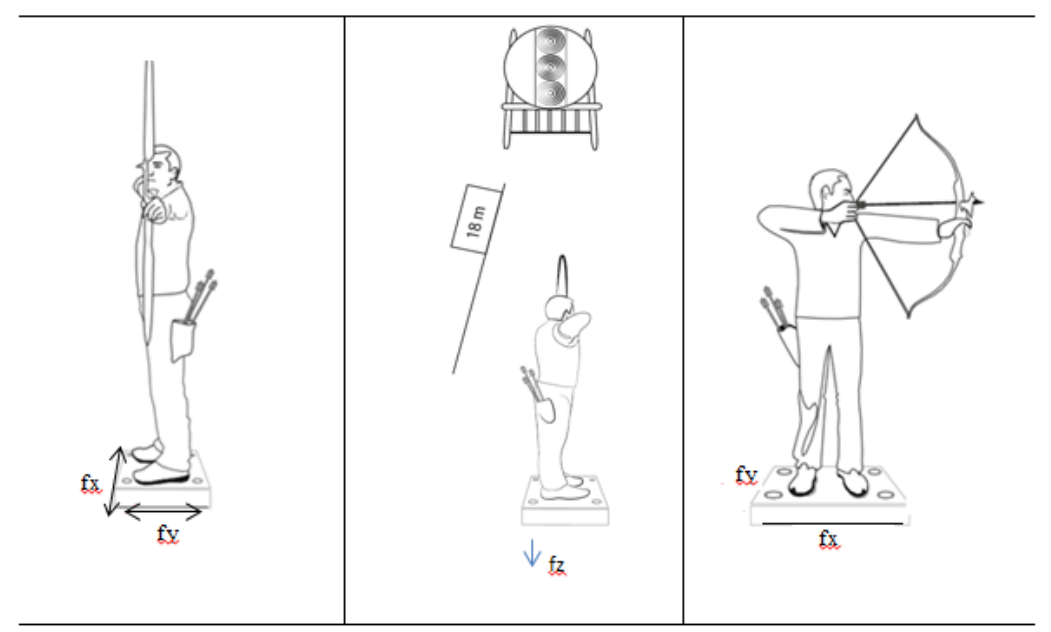

Picture 1. The schematic view of shooting direction.
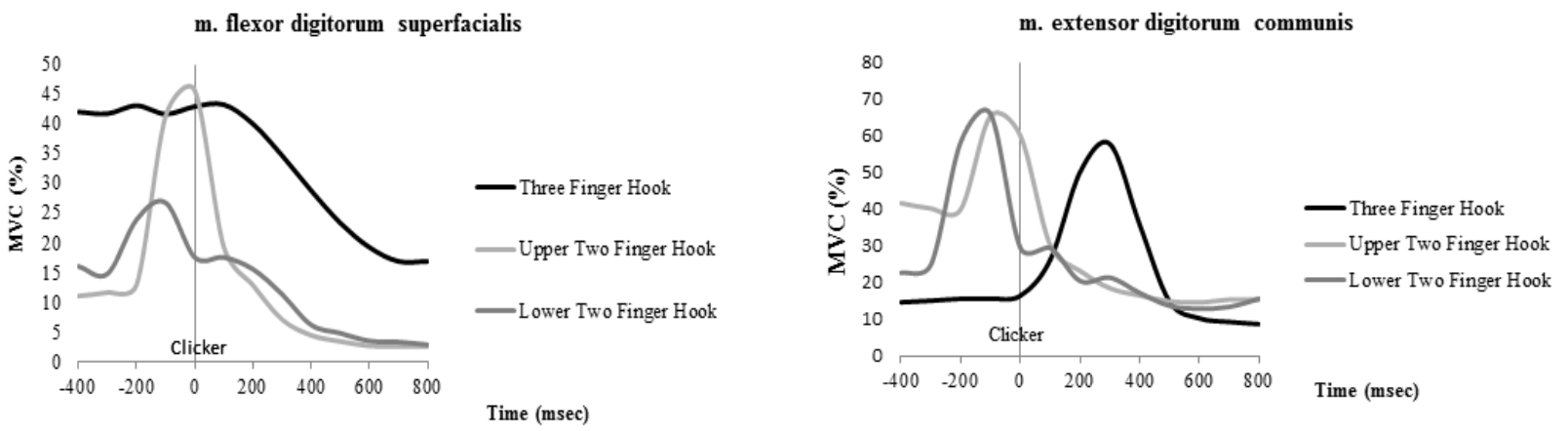

Figure 1. Drawing arm flexor and extensor muscles EMG values for different hook techniques.

\section{RESULTS}

\section{Muscular Activation Strategies}

In this study that was conducted on an elite archer, the muscular activation values of extensor, flexor, deltoid (anterior-middle and posterior), trapezius (upper-middle and lower), and pectorals major muscles were compared in terms of archer's three different hook techniques. According to this aim, muscular activation $400 \mathrm{msec}$ before and 800 msec after different releases of the sting and kinematic measures of elite archer was taken. The results for each muscle are displayed in Figures 1-3, showing the EMG activation levels of an elite archer.

The analyses of forearm flexor muscles showed that the three-finger hook demonstrated isometric contraction before the snap of the clicker and gradual relaxation just after the clicker's fall. However, the hook made by the second and third fingers had a different contraction/relaxation strategy compared with the three-finger hook. In the upper two-finger hook, there is a sudden contraction/relaxation of the flexor muscles just before the snap of the clicker. On the other hand, the lower two-finger hook has a contraction before the release and gradual relaxation after the snap As for the forearm extensor muscles, different finger placement techniques have created different muscular involvement strategies in which just after the fall of the clicker, extensor muscles suddenly contract to open the three finger hook, which result in the forward moment of the bowstring. The upper and lower two-finger hook techniques created extensor contraction just before the snap of the clicker (Figure 1).

In the finger hook, the deltoid anterior muscle had almost no contraction before the snap and a gradual contraction just after the snap of the clicker. This contraction corresponds with the sudden contraction of the forearm extensor, which may be related to the release of the weight in the upper and lower two-finger hook techniques had totally different strategy, which may not be related to early finding with archery release strategy. Kinesiology analyses of archery shooting during draw, full draw, aiming, and release phases require the horizontal abduction of the arm, which is carried by the deltoid middle and deltoid posterior. The deltoid middle has a stable contraction slope before and after the snap of the clicker. In the middle and posterior deltoid muscles, the three-finger hook contributed to the complete archery phases, synchronized with the other acting muscles. Deltoid posterior was very 
active before the snap of the clicker and gradually relaxed just before the snap of the clicker (Figure 2).

The most proximal point in archery shooting is the scapular joint. Archery coaches propose that the weight of the bowstring most the carried by the gleno-humeral and scapular joints. The scapular moment should be in the direction of the vertebra, called retraction and depression. Retraction and depression of the scapular joint are mostly caused by the middle trapezius and lower trapezius. The rhomboids muscles also contribute to these movements. The most proper use of the scapular joint is caused by the three-finger hook technique. The upper and lower finger hook technique has oscillations on the so called back muscles, which may cause lateral deflection of the bowstring (Figure $3)$.
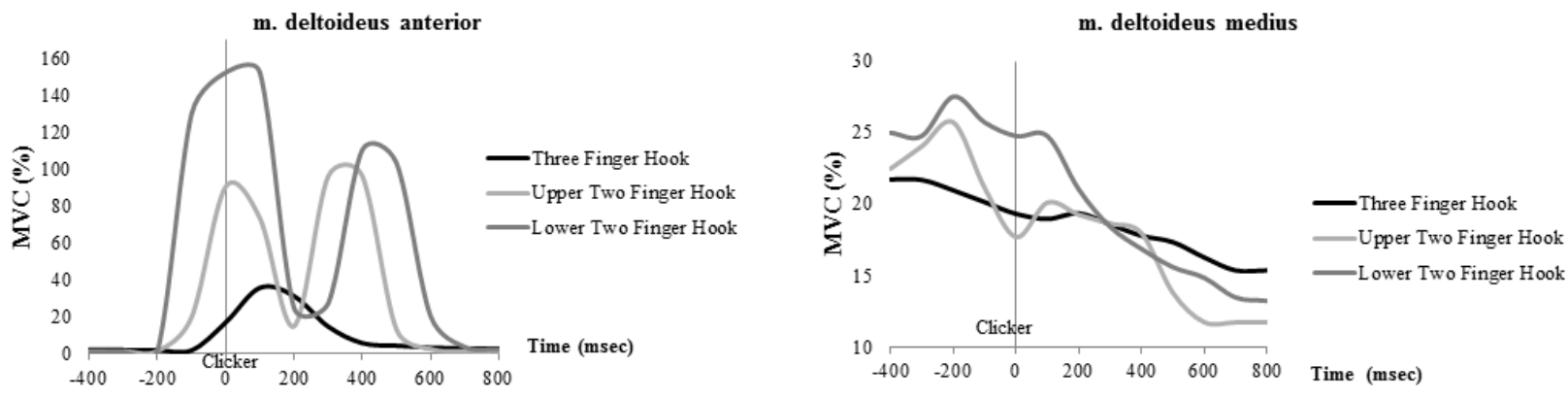

m. deltoideus posterior

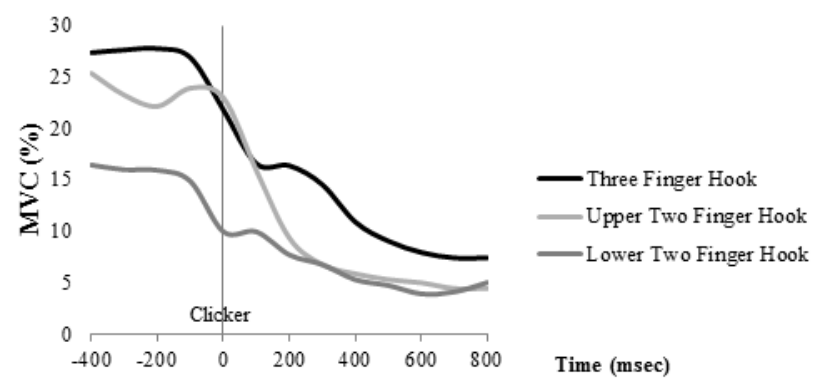

Figure 2. Drawing arm deltoid anterior-middle-posterior muscles EMG values for different hook techniques.
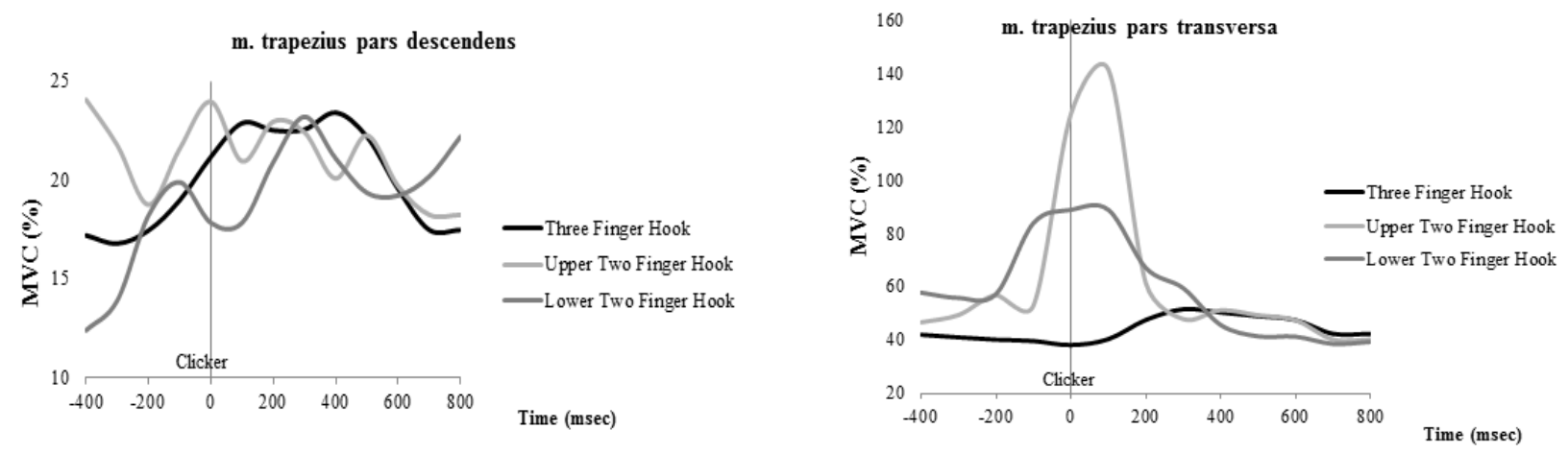

m. trapezius pars ascendens

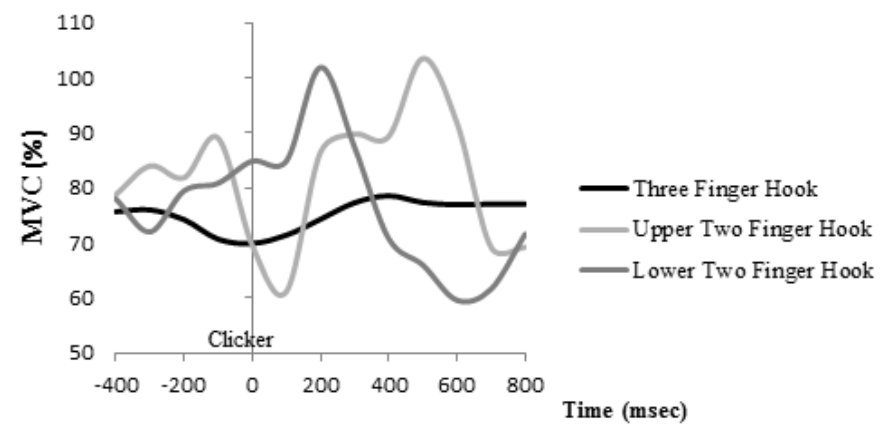

Figure 3. Drawing arm upper-middle-lower trapezius muscles EMG values for different hook techniques. 


\section{Postural control strategies}

The results for ground reaction forces are displayed in Picture 2-3, showing the anterioposterior (fy) and medio-lateral ( $f x$ ) forces and center of pressure sway (CoP sway) values for an elite archer.

The Picture 2 illustrates mediolateral or the sway in direction of target. The " $x$ " axis shows totally 1200 msec. minus 400 up to plus 800 , zero corresponds with the snap of the clicker. Before and after the snap of the clicker, the upper and lower two-finger hook showed a sharp increase in body sway in the anterior-posterior directions. The highest swa values were demonstrated with the lower two-finger hook technique, as compared to the upper two-finger hook and three-finger hook techniques. The three-finger hook technique showed the least sway. The " $y$ " axis shows the sway level in $\mathrm{cm}$, and minus indicates the pressure is higher in right foot. The highest level was achieved by the upper two-finger hook technique. The three-finger hook technique lean backward with a least value and demonstrated the closest level to zero.

The Picture 3 shows three different hook technique's mean CoP sway values. The highest CoP sway level was achieved with the upper twofinger hook technique. The three finger-hook technique illustrated a backward lean with the lowest value and was closest to zero. Before and after the snap of the clicker, the upper and lower two-finger-hook showed a sharp increase in body sway in the anterior-posterior directions. The highest sway values were demonstrated by the lower two-finger hook technique, as compared to the upper two-finger hook and three-finger hook techniques. The three-finger hook techniques had the least sway. The three different hook technique's mean CoP sway value was lower than both finger hook techniques and demonstrated the closest level to zero.
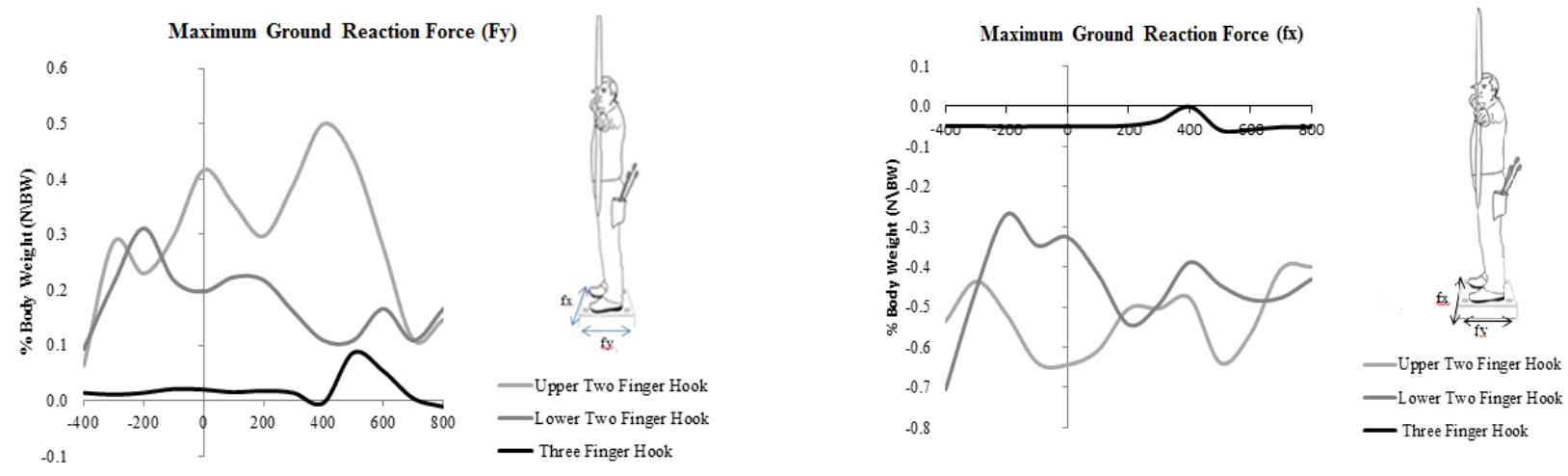

Picture 2. Maximum ground reaction forces (expressed as a percentage of body weight, BW) during three different hook techniques in the anterior-posterior axis (fy) and medio-lateral axis (fx).

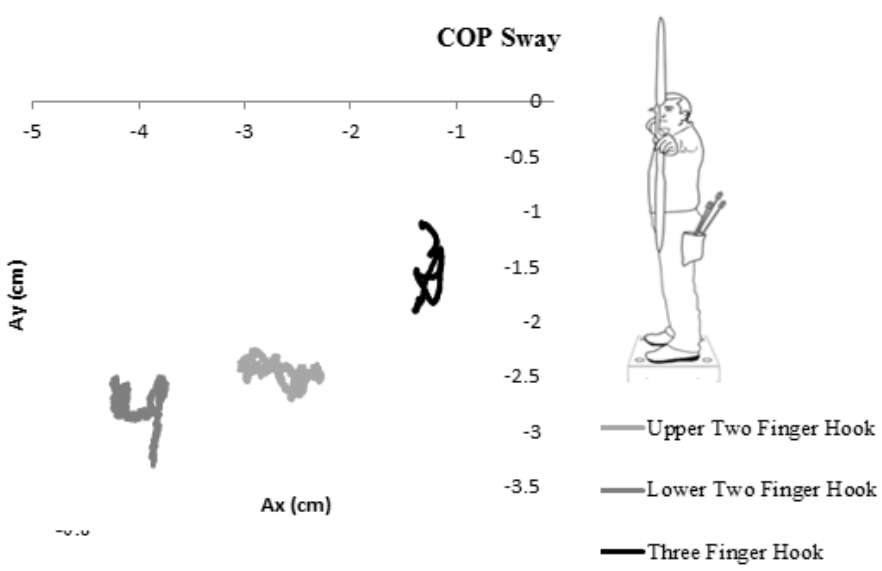

Picture 3. Three different hook technique's mean CoP from an $18 \mathrm{~m}$ distance (mean CoP of 3x4 arrow shots). 


\section{DISCUSSION}

The archery release is one of the most difficult fine motor abilities, which has a high impact on archery performance. Earlier findings have shown that before the snap of the clicker, the forearm flexor and extensor muscles contract almost equally to stabilize the range of motion in interphalangeal joints (7). In other words, archers have isometric contraction before the clicker's fall (4). Findings revealed that with different finger placement techniques on the bowstring, archers need to create three-finger hooks to achieve proper balance between the forearm flexor/extensor muscles. The finger hook affected isometric contraction before the snap of the clicker and caused sudden contraction of extensor, and gradual relaxation of the flexor muscles. This sudden forearm extensor contraction, which may be related to the release of the weight, in the upper and lower two finger hook techniques had totally different strategies, which may not related with earlier findings with archery release strategy (1-3). Kinesiology analyses of archery shooting during draw, full draw, aiming, and release phases require the horizontal abduction of the arm, which is carried by the deltoid middle and deltoid posterior. The deltoid middle has a stable contraction slope before and after the snap of the clicker. In middle and posterior deltoid muscles, the three-finger hooks contributed to the complete archery phases, synchronized with the other acting muscles. The deltoid posterior was very active before the snap of the clicker and gradually relaxed just before the snap of the clicker.

In conclusion; the most proximal joint is the scapular joint in archery and it is the center of the kinetic chain. During the full draw, aiming, and release techniques, scapular joint is supposed to retract and depress to achieve proper shooting technique in archery (8). By interpreting the findings of the current study, the most proper use of the scapular joint occurred during the three-finger hook technique.

\section{ACKNOWLEDGMENTS}

The current study has been supported by Anadolu University (Project number: Anadolu Uni./ BAP 1001S40).

\section{REFERENCES}

1. Ertan H, Kentel B, Tumer ST, Korkusuz F. Activation patterns in forearm muscles during archery shooting. Hum Mov Sci, 2003; 22; (1): 37-45.

2. Ertan H, Soylu AR, Korkusuz F. Quantification the relationship between FITA scores and EMG skill indexes in archery. J Electromyogr Kinesiol, 2005a; 15; (2): 222-227.

3. Ertan H, Kentel BB, Tumer ST, Korkusuz F. Activation pattern in muscles during archery shooting. Hum Mov Sci, 2005b; 22(1):37-45.

4. Hennessy MP, Parker AW. Electromyography of arrow release in archery. Electromyog Clin Neurophysiol, 1990; 30; (1); 7-17.

5. Leroyer P, Van Hoecke J, Helal JN. Biomechanical study of the final push-pull in archery. J Sports Sci, 1993; 11; (1): 63-69.

6. Mann DL, Littke N. Shoulder injuries in archery. Canadian Journal of Sport, 1989; 14(2), 85-92.

7. Martin PE, Siler W L, Hoffman D. Electromyographic analysis of bowstring release in highly skilled archers. J Sports Sci, $1990 ; 8 ; 215-221$.

8. Nishizono $H$, Nakagawa $K$, Suda $T$, Saito K. An electromyographical analysis of purposive muscle activity and appearance of muscle silent period in archery shooting. Jpn J Phys Fitness Sports Med, 1984; 33; (1): 17-26.

9. Rota S, Rogowski I, Champely S, Hautier C. Reliability of EMG normalisation methods for upper-limb muscles. Journal of Sports Sciences, 2013;31; (15), 1696-1704. 